\title{
Las TIC/TAC: subjetividades de los jóvenes universitarios en Montería.
}

\author{
Glenis Bibiana Álvarez Quiroz ${ }^{2}$ \\ Claudia del Pilar Vélez de la Calle ${ }^{3}$ \\ David Alberto Londoño-Vásquez ${ }^{4}$
}

Recibido: 05-01-2020

Aceptado: 19-05-2020

\section{Resumen}

El presente artículo analiza las subjetividades que se están generando en los jóvenes universitarios de Montería sobre las tecnologías y los tránsitos educativos que se dan allí. Para ello, se realizó un estudio de caso desde la perspectiva epistemológica del sujeto conocido. La recolección de la información se hizo a través de observación participante, grupos focales, relatos-historias de vida y narrativas digitales. Los participantes fueron 28 jóvenes de dos universidades, de entre 18 y 23 años, de diferentes pregrados. Entre las conclusiones se tiene que las TIC/TAC tienen un papel preponderante en la construcción de subjetividades de los jóvenes,

\footnotetext{
1. Este artículo es resultado de investigación de la tesis del Doctorado en Ciencias Sociales, Niñez y Juventud titulada Tecnologías de la información, comunicación, aprendizaje y conocimiento (TIC/TAC): comprensión de las subjetividades en jóvenes universitarios de Montería -tránsitos educativos en dos universidades—, que se realizó entre junio de 2013 y mayo de 2017.
}

2. Doctora en Ciencias Sociales, Niñez y Juventud. Docente del Departamento de Informática Educativa de la Facultad de Educación y Ciencias Humanas de la Universidad de Córdoba.

ORCID: http://orcid.org/0000-0002-6499-7124

Correo electrónico: gbalvarez@correo.unicordoba.edu.co

3. Doctora en Filosofía y Ciencias de la Educación. Directora del Doctorado de Educación, Universidad San Buenaventura - Cali. Investigadora sénior (Colciencias). ORCID: http://orcid.org/0000-0001-7014-047X

Correo electrónico: cpvelez@usbcali.edu.co

4. Doctor en Ciencias Sociales, Niñez y Juventud. Investigador de la línea estudios éticos, estéticos y comunicativos del grupo de investigación en Psicología Aplicada y Sociedad de la Institución Universitaria de Envigado. Docente de tiempo completo de la Institución Universitaria de Envigado.

Researcher ID: F-8907-2013.

ORCID: http://orcid.org/0000-0003-11110-7930

Correo electrónico: dalondono@correo.ive.edu.co 
pues podrían propiciar el desarrollo de un sujeto crítico con acceso a gran cantidad de información que eventualmente, y en ciertas condiciones, convertiría en conocimiento; no obstante, también podrían generar un sujeto pasivo a la información, consumidor y con pocos procesos intelectuales. En ese sentido, se están gestando unos sujetos replicados, copiados, trasladados; es decir, jóvenes ciberhomogeneizados, que están conectados por el ciberespacio pero que, a la vez, son homogeneizados a través de su vestimenta, construcción corpórea y formas de relacionarse.

Palabras clave: tecnologías de la información, comunicación, aprendizaje y conocimiento (TIC/TAC), jóvenes, subjetividades, educación superior.

\section{ICT/ACT: Young University Students' Subjectivities in Monteria}

\section{Abstract}

This paper analyzes the subjectivities that are being generated in the university students in Monteria on these technologies and the educational transits that occur with it. Therefore, a case study was carried out, from known subject epistemology perspective. Information collection was done by means of participant observation, focus groups, life relates-histories and digital narrations. Participants were 28 young students between 18 and 23 years from different undergraduate programs. Among conclusions, ICT/ACT have a preponderant role in the construction of subjectivities of young people; therefore, they could foster the development of a critical subject with access to a large amount of information that, in certain circumstances, would turn knowledge into knowledge; nevertheless, they also could generate a passive subject to information, consumer and with few intellectual processes. In this 
sense, some subjects are being replicated, copied, transferred, indeed, Young Cyber-homogenized, which are connected by cyberspace, but which are simultaneously homogenized through their dress, body building, and ways of relating.

Keywords: Information Technologies, Communication, Learning and Knowledge (ICT/ACT), youth, subjectivities, Higher Education.

\section{Introducción}

La inclusión de las tecnologías de la información y comunicación (TIC) en cada aspecto de la vida contemporánea las está convirtiendo en el centro de una nueva forma de vida de los sujetos sociales, especialmente de los jóvenes, lo cual está cambiando la manera de relacionarse con el mundo en muchos aspectos, económicos, políticos, culturales, sociales, educativos y de constitución singular, aproximándolos a identidades diversas y plurales logradas por sus prácticas de conectarse con el mundo. Es relevante mencionar que las tecnologías del aprendizaje y el conocimiento (TAC) tratan de orientar el desarrollo de las TIC hacia usos pedagógicos, con el objetivo de mejorar la formación y aprovechar los recursos que ofrecen dichas tecnologías. Es decir, las TAC van más allá de aprender a usar las TIC y apuestan por explorar estas herramientas tecnológicas al servicio del aprendizaje y de la adquisición de conocimiento (Lozano, 2011; Prat y Camerino, 2012).

Es fundamental una adopción más significativa de las TIC/TAC en los procesos educativos de la educación superior teniendo como principal valor el conocimiento, y en los que los jóvenes tengan oportunidades equitativas de incursionar con las mismas capacidades y posibilidades $y$, especialmente, empleando el contexto en el cual se desenvuelven.

Los sujetos de la educación de la modernidad ya no son los mismos que los de la época contemporánea (Roy, 2012; Abdel- 
Aziz, Abdel-Salam y El-Sayad, 2016). Por tanto, la inclusión de las TIC/TAC en la vida de los sujetos y sus ámbitos ha creado un hito en el que la adopción es formal pero no uniforme y mucho menos consciente (Aguilar y Said, 2010; Álvarez y Vélez, 2014). Formas de ser, enseñar y aprender se confunden en los espacios de formación universitaria, con el resultado de que los docentes, la mayoría de las veces, quedan en el último lugar de estas transformaciones, siendo superados por los estudiantes en velocidad de apropiación de la información y del manejo de las mediaciones mencionadas (Bennett, Maton y Kervin, 2008; Fuente, 2017).

Teniendo cuenta lo anterior, surge el interés por comprender las subjetividades generadas por un grupo de jóvenes de dos universidades de Montería a partir de las TIC/TAC, e identificar los tránsitos educativos emergentes por medio de interpretaciones de las narrativas digitales, a través de grupos focales e historias de vida sobre corporeidad, manejo del espacio y tiempo como sujetos de formación.

\section{Referentes teóricos}

En este apartado se presentan brevemente los conceptos de subjetividad juvenil digital y TIC/TAC, los cuales sirvieron como marco de comprensión de los resultados obtenidos.

\section{Subjetividad juvenil digital}

Con las TIC/TAC, el espacio de un sujeto determinado ya no se localiza en un territorio determinado, sino que a través de los entornos virtuales es posible ejercer su subjetividad desde diferentes espacios (Aguilar y Said, 2010; Goktas y Demirel, 2012). Estos entornos ofrecen nuevas formas de estar en el mundo, socializarnos y comunicarnos (Castells et al., 1986; Martín-Barbero, 2011). Se está gestando un nuevo sujeto con acceso a gran cantidad de medios 
de información y de posibilidades de relación con otros a través de redes y otros dispositivos, lo cual ha devenido en algo natural (Erazo y Muñoz, 2007; Collins, Welsh y Furman, 2009). Los entornos virtuales de socialización se reconocen como nuevas instancias de comunicación, y de generación de diferentes códigos comunicativos y subjetividades (Furman y Buhrsmester, 2009; Gardner y Davis, 2014).

Por tanto, es posible pensar que el sujeto es un resultado de las dinámicas sociales y culturales que lo circundan, nace ligado al consumo y a la voluptuosidad (Deleuze, 2002; DeLanda, 2006). El sujeto joven está inmerso en una serie de nuevas prácticas culturales en las que la comunicación y la interacción con las tecnologías es fundamental; son nuevas prácticas para relacionarse en el mundo (Muñoz, 2007; Giraldo-Gutiérrez, Zúñiga-Miranda, LondoñoVásquez y Sánchez-Ceballos, 2018). Al respecto, Foucault (1988) muestra cómo los modos culturales configuraron las subjetividades; en este caso las prácticas de los jóvenes en las que se conectan a través de imágenes, signos, textos y otras representaciones audiovisuales; de allí que sus actividades diarias estén estructuradas alrededor del uso de los medios de comunicación y se configuren como modos culturales (Duggan, Ellison, Lampe, Lenhart y Madden, 2015; Álvarez y Vélez, 2015). Por ello, se está produciendo una acelerada transformación en las formas de vida de los jóvenes potenciadas con el uso de las tecnologías, en contraste con los modos que le precedieron antes de la masificación de estas mediaciones tecnológicas (Álvarez y Blanquicett, 2015; Suárez, Vélez y Londoño, 2018).

Debido a los constantes cambios, se está generando una subjetividad juvenil digital (Latour, 2007), pues hay una interacción primordial entre los humanos y no humanos que, enmarcada en el contexto de las TIC/TAC, se constituyen en modos culturales y educativos propios que influyen como agenciamientos en las configuraciones subjetivas.

La concepción del sujeto ya no es la misma y cabe preguntarnos si en realidad hay un sujeto. Baudrillard (1989) plantea la idea de 
sujeto fractal vinculada a los medios y a la pantalla, en donde se vislumbra una nueva subjetividad. Se están gestando unos sujetos replicados, copiados, trasladados, que en el presente artículo se denominan jóvenes ciberhomogeneizados para hacer referencia a los jóvenes que están conectados por el ciberespacio pero que, a la vez, son homogeneizados a través de su vestimenta, peinados, formas de relacionarse, generando así "nuevas identidades que se alimentan, actualizan y regeneran con cada nuevo movimiento y acción nuestra, traducidos a dato" (Callén y Tirado, 2006, p. 34).

\section{Las TIC/TAC}

Las TIC posibilitan nuevos modos de percepción, nuevas sensibilidades y lenguajes que, dadas las circunstancias, evidencian su enorme capacidad de penetración y que hace difícil imaginar situaciones en las que, de alguna manera, las TIC no estén presentes (Álvarez y Vélez, 2014). Cuando las TIC se orientan hacia usos pedagógicos, con la finalidad de mejorar la formación y aprovechar los recursos que ofrecen dichas tecnologías, se denominan TAC. AI respecto, Vivancos (febrero de 2009) propone que las TAC equivalen al e-learning y a la gestión del conocimiento, lo cual permite plantear el cambio del aprendizaje de la tecnología por el de aprendizaje con la tecnología, con lo cual se orienta íntegramente al desarrollo de competencias metodológicas fundamentales, como el aprender a aprender (Lozano, 2011), permitiendo adquirir la "intuición digital", es decir, la metodología y las herramientas necesarias para que se le dé sentido a lo aprendido (Reig, 2012).

Al parecer, el campo educativo no está exento de este impacto en menor o mayor grado (Ortega, Rey y Sánchez, 2012; Scherer y Hatlevik, 2017), y es fundamental preparar a las nuevas generaciones para que estén a la altura de los nuevos procesos de socialización que se están generando con las TIC (Lee y Ma, 2012; Sánchez, Muñoz y Ortega, 2015), en las cuales no solo prima el conocimiento (Lupton, 2014; Whelan et al., 2016), sino también la economía (Dinero, 2017; Yang, Ren y Adomavicius, 2018), las comunicacio- 
nes (Renó, 2007; Wilson, Gosling y Graham, 2012), la educación (Ertmer, 2005; Papastergiou, Gerodimos y Antoniou, 2011), ligadas a la innovación (Hartley, 2008; Yunis, Tarhini y Kassar, 2018).

Es por ello que las TIC/TAC ofrecen la posibilidad de realizar actividades como experimentos virtuales, facilitan nuevas modalidades de trabajo colaborativo y permiten tanto la profundización como el refuerzo personalizados de algunos aprendizajes. Existe un amplio abanico de recursos TIC/TAC disponibles en internet, lo que abre nuevos horizontes a la programación didáctica y a la adopción de estrategias orientadas al aprendizaje basadas en problemas y situaciones reales.

\section{Perspectiva epistemológica y metodológica}

La perspectiva epistemológica de la presente investigación se fundamentó en la epistemología del sujeto conocido (Vasilachis, 2006), que implica reconocer su capacidad para incidir en la transformación de sus propios contextos. Se le muestra como un sujeto políti$\mathrm{co}$, una subjetividad que construye poder, con una mayor autonomía y capacidad de decisión sobre sus procesos educativos.

La perspectiva metodológica tuvo una orientación cualitativa; este tipo de investigación facilita el aprendizaje de las culturas y proporciona al investigador formas de examinar el conocimiento, el comportamiento y los artefactos que los participantes comparten y usan para interpretar sus experiencias (Londoño, Olave, Jaime y Losada, 2018). Se trabajó con el estudio de caso, ya que este tipo de investigación permite analizar un fenómeno contemporáneo dentro de su contexto real (Yin, 1984).

En el presente estudio se asume como población de referencia a los estudiantes de dos universidades ubicadas en la ciudad de Montería. La muestra estuvo constituida por 28 jóvenes. Para su selección se tuvieron en cuenta los siguientes criterios: 
- Pertenecieran a la Universidad de Córdoba (Unicórdoba) o a la Universidad Cooperativa de Colombia (UCC) - Sede Montería.

- Igual número de jóvenes de ambas universidades (14).

- De edades entre los 18 y 23 años.

- Los jóvenes debían estar cursando entre tercer semestre y décimo semestre. No se trabajó con jóvenes de primer y segundo semestre debido a que se necesitaba que tuvieran al menos un año en la universidad.

- Se buscó que los estudiantes pertenecieran al menos a cuatro programas diferentes en ambas universidades, para tener diversidad de perfiles. En Unicórdoba, los jóvenes pertenecen a los programas: Licenciatura en Informática y Medios Audiovisuales, Licenciatura en Lengua Castellana, Licenciatura en Música, Ingeniería Mecánica, Bacteriología, Licenciatura en Ciencias Sociales. En la UCC - Sede Montería, los estudiantes pertenecen a los programas de Psicología, Ingeniería de Sistemas, Derecho y Administración de Empresas.

Por otro lado, con respecto a las técnicas de recolección de información y el corpus recolectado, la investigación trabajó con observación participante (Jorgensen, 2015) 512 relatos-historias de vida (Cardona, Valencia, Duque y Londoño, 2015) ${ }^{\circ}, 12$ narrativas digitales (Amador, 2013) y y 6 grupos focales (Stewart y Shamdasa-

\footnotetext{
5. Una de las autoras fungió como docente de los participantes en ambas universidades. Esto le permitió no solo observar a los participantes en los diferentes momentos de la aplicación de las técnicas, sino también dentro de la cotidianidad del aula. En relación al tema de lo participante, la autora, dentro de sus cursos, también proponía lecturas y autores sobre estos temas para la discusión.

6. Se invitó a los 28 participantes a realizar un relato autobiográfico en video llamado Mi vida y las TIC. Solo 12 aceptaron la invitación. Allí ellos enfatizaron en su vida universitaria, en el tipo de herramientas utilizadas, el motivo de uso, la intensidad horaria aproximada y otros actores involucrados en esta (amigos, familiares, entre otros). Estos relatos fueron transcritos.

7. Los 12 participantes que realizaron los relatos autobiográficos recibieron una capacitación por parte de una de las investigadoras en edición de videos. Esto les permitió recrear sus relatos iniciales y producir narrativas digitales, los cuales estuvieron a disposición del público durante el año 2016.
} 


\author{
ni, 2014). El corpus fue analizado a través de análisis de contenido \\ (González, 2000)', empleando una matriz de coherencia metodoló- \\ gica (Duriau, Reger y Pfarrer, 2007) ${ }^{10}$ para identificar las categorías \\ a analizar.
}

\title{
Análisis y resultados
}

\section{A partir del empleo de la matriz de coherencia metodológica, se identificaron 3 categorías y 15 subcategorías, así:}

Tabla 1. Categorías y subcategorías.

\begin{tabular}{|l|l|}
\hline $\begin{array}{l}\text { Las tecnologias de la } \\
\text { información y comunicación, } \\
\text { el aprendizaje y el } \\
\text { conocimiento }\end{array}$ & $\begin{array}{l}\text { - ¿Qué son las TIC? } \\
\text { - Aspectos negativos de las TIC } \\
\text { - Las TIC en la universidad práctica } \\
\text { - Las TAC en la universidad }\end{array}$ \\
\hline Tránsitos educativos & $\begin{array}{l}\text { - Profesores y TIC/TAC } \\
\text { - Escrituras } \\
\text { - Prácticas y herramientas usadas } \\
\text { - Prácticas no éticas con las TIC }\end{array}$ \\
\hline $\begin{array}{l}\text { - Nativos digitales-inmigrantes digitales } \\
\text { - Discursos y terminologías } \\
\text { - Relaciones interpersonales } \\
\text { - Compras y ventas a través de la web } \\
\text { - TIC y tiempo } \\
\text { - TIC y espacio } \\
\text { - Cuerpo y TIC }\end{array}$ \\
\hline
\end{tabular}

Fuente: elaboración propia de los autores.

8. Los grupos focales se realizaron en seis momentos diferentes durante los años 2015-2016. Esto se dio con el propósito de que los 28 participantes pudieran ser parte de alguno de los grupos focales. Se realizaron tres en Unicórdoba y otros tres en la UCC. El guion para los grupos focales surgió de la revisión de literatura realizada, el análisis de los 12 relatos y de las narrativas digitales. Este guion fue validado por 5 expertos antes de su aplicación. Cada grupo focal por universidad tuvo una temática previamente definida: 1) influencia de las TIC/TAC en los espacios de formación universitaria, 2) cambios generados por las TIC/TAC en la corporeidad, manejo de espacio y tiempo como sujetos de formación, y 3) discursos y prácticas sobre la influencia de las TIC/TAC en la construcción de las subjetividades en jóvenes universitarios.

9. El análisis de contenido representa el significado de un fenómeno estudiado, donde se activa un bloque de información integrado al contexto o a la estructura que le confiere el informante clave, de acuerdo a la experiencia en el tema tratado (González, 2000). Se interesa por las acciones humanas a través de las relaciones descriptivas que el investigador hace de los datos (Campos y Mújica, 2008).

10. Con esta matriz se evaluó y presentó una visión panorámica del proceso. Se relacionaron los siguientes componentes: tema, campo de estudio, preguntas de investigación, estado del arte, categorías, objetivos, enfoques, diseño metodológico, técnicas, instrumentos. La matriz apoyó el proceso de análisis y dio una visión general del diseño metodológico de la investigación, a partir del análisis de cada objetivo. Partiendo de esto se procedió a realizar el análisis de contenido. 
El análisis de contenido permitió corroborar que estas estuvieran presentes en el corpus, puesto que fueron emergiendo allí".

Las tecnologías de la información, comunicación, aprendizaje y conocimiento (TIC/TAC)

\section{¿Qué son las TIC?}

Hoy en día las TIC se han extendido ampliamente en la vida cotidiana de una manera sin precedentes (Curran, Fenton y Freedman, 2016). Se presta atención a las TIC sin tener en cuenta el aspecto significativo de socialización que implican (Brandtzæg, Heim y Karahasanović, 2011). Con la incursión masiva de internet, así como de las aplicaciones de teléfonos inteligentes y las redes sociales digitales, las personas se relacionan socialmente privilegiando la conexión a través de espacios virtuales en detrimento del encuentro físico en espacios públicos o privados.

Entre las definiciones de TIC que proporcionaron los participantes una de ellas señala que son

herramientas que facilitan el acceso a toda la información; las TIC facilitan a las personas el acceso a la información, lo cual ha permitido que en la cotidianidad algunas cosas sean más sencillas, como para un trabajo académico; además, permiten tener comunicación con personas que estén a distancia o si necesitamos hablar con personas inmediatamente; por ello, dentro de nuestra cotidianidad nos hacen las cosas más fáciles. (Relato de la historia de vida de MU2MA) ${ }^{12}$

\footnotetext{
11. El análisis de contenido permite a los investigadores analizar las construcciones sociocognitivas y perceptivas que son difíciles de estudiar a través de métodos de archivo cuantitativos tradicionales. Es una técnica de investigación utilizada para hacer inferencias replicables y válidas interpretando y codificando material textual (Duriau, Reger y Pfarrer, 2007).

12. Con el ánimo de mantener el anonimato de los 28 participantes, los relatoshistorias de vida, los textos transcritos de los grupos focales y las narraciones digitales utilizadas en el análisis fueron codificados. En esta codificación, $M$ es para mujer y $\mathrm{H}$ para hombre; luego, U1 y U2 para las dos universidades de donde provienen: Unicórdoba y Cooperativa - Montería, respectivamente. Finalmente, las dos últimas letras corresponden a las iniciales de sus nombres de pila. Además, por cuestiones de espacio en la publicación, solo se mencionan los apartados más relevantes del corpus.
} 
En ese sentido, es de resaltar la amplia gama de recursos que ofrece internet, posibilitando nuevas formas de comunicación y de relaciones de aprendizaje (Dede, 2000; Genlott y Grönlund, 2016). Entre las características de las TIC citadas por varios autores, se encuentran: la interactividad, la innovación, la inmaterialidad, la digitalización, la calidad de imagen y sonido, y la interconexión y diversidad (Castells et al., 1986; Gisbert, 1992; Gutiérrez y Orozco, 2007).

\section{Aspectos negativos de las TIC}

La introducción de las TIC en el sistema educativo en las últimas dos décadas no ha tenido el impacto que se esperaba, a pesar de su potencial (Sandholtz y Reilly, 2004). Por el contrario, ha puesto en evidencia la ausencia de un compromiso significativo y verificable por parte de los docentes (Jaramillo y Ruiz, 2009).

Entre los aspectos negativos manifestados, está el hecho de que algunos docentes no están formados ni capacitados para un buen uso de dichas herramientas en el contexto educativo, en donde se perjudica - de cierta manera- la educación, ya que los estudiantes no le dan el uso correcto. (Relato de la historia de vida de MU1CE)

Los profesores son el elemento clave para introducirlas en la práctica educativa, proveyendo a los estudiantes de productos ágiles, agradables, amigables, de contenido significativo. Sin estos agentes esenciales, la integración de recursos tecnológicos nunca se llevaría a cabo, pues parece ser que en el sistema educativo se asume que la responsabilidad es esencialmente de los profesores (Ertmer, 2005). En el fondo se evidencia la ausencia del compromiso de la institución educativa (Villalobos, Flórez y Londoño, 2017).

\section{Las TIC en la universidad}

En cuanto a la educación superior, las TIC son consideradas herramientas fundamentales; se utilizan en muchas áreas, tales como: el 
desarrollo de materiales del curso (Lupton, 2014), la entrega y la posibilidad de compartir contenidos (Hattangdi y Ghosh, 2008), y fomenta la comunicación entre los estudiantes, los profesores y el mundo exterior (Sandholtz y Reilly, 2004). Algunos de los estudiantes manifestaron su inquietud:

La universidad no está aprovechando todas las potencialidades de las TIC. (Relato de HU1A en un grupo focal)

Los estudiantes usan las TIC en la universidad dependiendo de las circunstancias y el momento. Muchas veces, se convierten en distractores ya que la usan para las redes sociales y no para prestarle atención a las clases. (Relato de HU2JF en un grupo focal)

Es relevante profundizar de manera continua en el uso de las TIC/TAC en procesos educativos en la universidad cuyo principal valor sea el conocimiento, y en los que los jóvenes y los docentes tengan oportunidades equitativas de incursionar con las mismas capacidades y posibilidades $y$, especialmente, aprovechando el contexto en el cual se desenvuelven. El usuario de las tecnologías se inscribe en un contexto de interacción crítica con los lenguajes, prácticas y productos tecnológicos (Suárez, Vélez y Londoño, 2018).

\section{Las TAC en la universidad}

Las TAC tratan de conocer y explorar los posibles usos didácticos que las TIC tienen para el aprendizaje y la docencia. En otras palabras, las TAC van más allá de aprender a usar las TIC y apuestan por explorar estas herramientas tecnológicas al servicio del aprendizaje y de la adquisición y gestión del conocimiento; en realidad lo que se plantea es cambiar el aprendizaje de la tecnología por el aprendizaje con la tecnología, enfoque orientado totalmente al desarrollo de competencias metodológicas fundamentales, como el aprender a aprender (Lozano, 2011).

Al respecto, un participante propone que: 
algunas aplicaciones que pueden usar los profesores como TAC, pueden ser Moodle - crear cursos virtuales-, Joomla! —crear páginas web—, Wordpress — crear blogs-. (Relato de HU1JA en un grupo focal)

De esta manera, se realizaría un ejercicio general y básico por parte de los estudiantes que debería consolidarse hasta dar lugar a nuevos y creativos usos de las tecnologías más allá de las plataformas y aplicaciones existentes. Se trata de evidenciar la importancia de la decisión política y administrativa de las entidades de educación superior de comprometerse directamente con el soporte, apoyo y fortalecimiento de las TAC a lo largo y ancho de las instituciones, no dejando en responsabilidad exclusiva del docente dicho proceso.

Con las TAC se busca incidir especialmente en la metodología de enseñanza, en los usos creativos y efectivos de la tecnología, y no exclusivamente en asegurar el manejo de una serie de herramientas informáticas. Se busca conocer e indagar los usos didácticos que las TIC tienen para el aprendizaje y la docencia y avanzar en sus posibles desarrollos como TAC.

\section{Tránsitos educativos}

Los tránsitos educativos se refieren a los cambios que se dan a lo largo de la vida académica en los distintos niveles educativos, por los efectos causados por distintos entornos, metodologías de trabajo y realidades (Pié Balaguer, 2014). En otras palabas, vivir en tránsito, con cambios constantes de las personas y sus relaciones sociales, parece conducir a una deconstrucción más radical que la practicada por las teorías de la sospecha sobre la subjetividad y la conciencia (García Canclini, 1997).

En especial, se consideran tránsitos educativos los nuevos vínculos que están emergiendo con los usos cognitivos y sociales de las TIC. Dicho de otro modo, los trayectos desiguales y combinados 
que suceden cuando se están apropiando social, cognitiva y culturalmente nuevos modos y formas de aprender y de enseñar.

\section{Profesores y TIC-TAC}

Uno de los mayores desafíos que enfrentan los centros de enseñanza superior en el siglo XXI no son las nuevas tecnologías en sí, sino la capacidad de los educadores para integrar las tecnologías educativas (Watty, McKay y Ngo, 2016). Mientras que la tecnología ofrece nuevas oportunidades y beneficios para los educadores en su práctica docente, un número significativo de profesores son resistentes a la adopción de nuevas tecnologías.

Algunos estudiantes consideran que los docentes no están suficientemente capacitados en el uso de algunas tecnologías, puesto que,

en su mayoría, los profesores no usan herramientas para desarrollar una clase más dinámica, todo lo hacen de manera magistral mediante solo el habla y, en algunos casos, llevan libros a clase y los leen. (Relato de HU2R un grupo focal)

Otros plantean que aunque algunos docentes usan TIC en sus clases, muchas veces no lo hacen de la mejor forma:

Aunque las diapositivas son una buena herramienta, algunos docentes saturan con demasiada información y muchas veces los estudiantes no alcanzan a anotar lo más importante. (Relato de HU1JD en un grupo focal)

Parece ser que el profesor universitario se enfrenta hoy a nuevos retos pedagógicos, dado que tiene que diseñar entornos de aprendizaje que respondan a las necesidades y requerimientos cambiantes de los estudiantes - que muchas veces expertos en tecnología- y tienen que integrar las TIC en sus cursos para flexibilizar los servicios y contenidos educativos. 


\section{Escrituras}

Con las herramientas digitales de hoy en día -como los teléfonos inteligentes y las tabletas-, la lectura y escritura ya no tienen interfaces separadas para las dos prácticas (Taipale, 2015). Simultáneamente, las pantallas táctiles se utilizan para llevar a cabo ambas prácticas (Emerson, 2014). Además, las nuevas plataformas de medios interactivos han surgido reclamando nuevos tipos de habilidades de comunicación. Son estas nuevas interfaces y el ascenso a las plataformas de medios de colaboración los que han cambiado las habilidades de solo lectura a las de leer y escribir al mismo tiempo (Hartley, 2008).

Entre las manifestaciones subjetivas de los estudiantes con respecto a la escritura se encuentran:

Escribir a lápiz es algo que se le ha olvidado un poco, la ortografía y la caligrafía cambian, ya que él no está acostumbrado a escribir por medio físico, y menos en la universidad. (Relato de HU1S en un grupo focal)

Tengo muchos errores ortográficos, ya me he acostumbrado al autocorrector del celular y del computador, por lo cual, se me olvidan los signos de puntuación. (Relato de la historia de vida de HU1JJ)

En cuanto a las herramientas digitales, la escritura está mediada por el hardware (la máquina de escribir o el teclado) y, cada vez más, también por el software (por ejemplo, sistemas de predicción de texto, herramientas de anotaciones). Para la lectura y la escritura, aparte de la capacidad de presionar un botón o tocar una pantalla, también se tienen que dominar algunas otras habilidades tales como la forma de cambiar una línea, la forma de conectarse a un computador y cómo utilizar un procesador de textos. Por tanto, con los cambios de las bases materiales de la lectura y la escritura, las habilidades también tenían que cambiar (Shove y Pantzar, 2005). 


\section{Prácticas y herramientas usadas}

La expansión de las TIC permite a las personas recibir, procesar y crear más información que en cualquier momento de la historia humana (Massaro, 1993). Los consumidores de los nuevos medios de comunicación se han convertido en productores, que no solo leen los contenidos digitales, sino que también crean contenidos (Taipale, 2015).

Entre las herramientas y aplicaciones más usadas por los estudiantes universitarios de Montería figuran los computadores portátiles, los celulares y las tabletas, en la forma como lo dan a conocer en los siguientes apartados:

Me gusta usar la tablet porque es más fácil de tenerla en las manos para mayor accesibilidad; si salgo para cualquier parte o si necesito movilizarme, utilizo, en general, las redes sociales, tengo Facebook, MSN, los buscadores. También para acceder a las aplicaciones de la universidad como Powercampus, donde verifico mis notas, manejo los cursos en los que me inscribiré, entre otras cosas. Ya el computador es como para trabajos escritos, para otro tipo de cosas fuera del contexto académico. (Narrativa digital de HU1LD)

Las redes sociales son las herramientas más usadas por los estudiantes:

Las redes sociales nos permiten comunicarnos con personas que están lejos, lo que facilita la comunicación. (Narrativa digital de MU2MC)

El estudio de las redes sociales ha atraído mucho interés por parte de la comunidad en los últimos años (Whelan, Teigland, Vaast y Butler, 2016). Facebook es la plataforma más popular de redes sociales; pero varias aplicaciones de redes sociales han comenzado a ganar popularidad entre los adultos jóvenes; por ejemplo, Twitter, Instagram, Pinterest, Snapchat (Duggan, Ellison, Lampe, Lenhart y Madden, 2015). Al respecto, 
Me gustan los editores de fotos, los reproductores de música —no para descargarla sino como una radio-, también las aplicaciones que todo el mundo tiene, Facebook, WhatsApp, Snapchat, Instagram y Twitter. (Narrativa digital de MU2MA)

Twitter es una de las plataformas de redes sociales más populares para los estudiantes; se ha encontrado que algunos profesores la utilizan para compartir información, recursos y medios propios de su enseñanza (Lupton, 2014). Por ejemplo, los estudiantes han demostrado que usan Twitter para pedir y ofrecer ayuda a otros y contribuir con sus opiniones a través de hashtags ${ }^{13}$ (Mandavilli, 2011).

Otra de las herramientas usadas por los estudiantes son los blogs:

Tengo un blog sobre arte, dibujo, música, escritura, y en general, sobre cuestiones artísticas. También tengo uno sobre literatura en especial, lo que tiene que ver con ortografía, fonética, narración. Tuve la oportunidad de grabar una canción con un amigo, lo que me ha dado grandes satisfacciones. (Narrativa digital de HU1LD)

Los blogs fomentan la participación en el proceso de aprendizaje, ya que cambia el flujo y el contenido de los cursos de una pista tradicional de aprendizaje a un lugar más interactivo (Goktas y Demirel, 2012). De acuerdo con algunos estudios, con ellos los estudiantes se vuelven más activos y más responsables de su propio trabajo, se estimula la curiosidad, tienen el potencial para aumentar el interés y compromiso de los estudiantes en el aprendizaje y pueden ser utilizados para animar a los estudiantes a explorar activamente los conceptos del curso (Papastergiou, Gerodimos y Antoniou, 2011; Pineda y Tamayo, 2016; Suárez-Palacio, Vélez-Múnera y Londoño-Vásquez, 2018).

13. Un hashtag significa que se está usando un conjunto de caracteres - p. ej. una palabra - para destacar una información, para agrupar una temática o un conjunto de conversaciones, con el signo \# precediéndolo, por ejemplo \#hashtag. 
También manejan aplicaciones como Google Drive (que sirve para crear, subir documentos en línea y luego compartirlos). Les gusta jugar en línea en sus ratos libres; entre las aplicaciones más usadas están los juegos para desarrollar la mente como Sudoku, simulaciones, juegos en línea y para competir con personas de otros lugares; también usan juegos de lucha libre, y billar online, donde hacen apuestas.

Para labores académicas se usan las aplicaciones de la universidad como Powercampus (plataforma de notas, cursos, historial académico de Unicórdoba) y Timonel (plataforma de notas, cursos, historial académico de la UCC). También se usan bases de datos como Psicodoc, Redalyc, Scielo, ProQuest, Ebrary, Google Académico, y plantean que en varias ocasiones no se confían de la información que les dan los buscadores normales como Google y prefieren buscar en bases de datos.

Se evidenció, a través de los grupos focales y narrativas digitales, que en su mayoría los estudiantes se sienten a gusto con las herramientas que usan a diario, y esto les ha ayudado a afianzar algunos conceptos que trabajan en clases y a estar al día con sus relaciones personales.

\section{Prácticas no éticas con las TIC}

Las TIC han facilitado algunas prácticas no éticas en los entornos universitarios, como el plagio, sobre lo cual expresan:

Lastimosamente es común que, durante los exámenes, algunos estudiantes le toman fotos a la hoja, y se la envían a algún compañero por fuera para que realicen el examen, por lo cual pagan entre 20 o 30 mil pesos, dependiendo de la complejidad. Es muy común, sobre todo en los que estudian ingeniería, administración, física, química, en lo que tiene que ver con cálculos, y en general, en procesos matemáticos. (Relato de HU1JA en un grupo focal) 
Cuando nos envían trabajos escritos, generalmente busco en la página El Rincón del Vago para hacerlos, copio, y pego, algunas veces cambio palabras. Eso lo hacemos casi todos los estudiantes del salón. (Relato de la historia de vida de HU2R)

El plagio es una de las prácticas no éticas con las TIC, y desde la educación no debe tomarse a la ligera; la comprensión del concepto de plagio utilizando las TIC es todavía insatisfactorio (Scanlon, 2003; Boisvert e Irwin, 2006). Se debe hacer un esfuerzo continuo para inculcar la comprensión entre los estudiantes sobre el plagio, para evitar su participación en él en el futuro, ya que no solo se afecta la imagen de ellos como estudiantes sino también la imagen de la institución.

El tema de reflexión alude a la escasa valoración del conocimiento y el saber como proceso que se asume, se disfruta, se enfrenta y se resuelve, como una gestión del conocimiento (Arias, Londoño, Betancourt y Restrepo, 2017) en la cual los estudiantes como individuos, los docentes como investigadores o diseminadores de conocimientos y la institución educativa se encuentran lejos de consolidar dicha valoración (Baron, 2008).

La humanidad vive una época de cambios vertiginosos; la globalización se nota claramente en el apogeo de las TIC, lo que lleva a nuevos desafíos que trasladan el papel de los medios de comunicación dentro de una sociedad de saberes participativos, globales y cada vez más homogeneizados (Bauman, 2017). Los jóvenes de la época contemporánea deben vivir con cambios mucho más rápidos que los jóvenes de otras épocas. Todo ello se ve reflejado en la cultura mediática, la cual tiene el potencial de crear tránsitos educativos que ofrecen viabilidad para superar los logros de las formas institucionalizadas de educación. 


\section{Subjetividades juveniles digitales}

En la categoría de subjetividades juveniles digitales emergieron siete subcategorías, a saber: nativos digitales-inmigrantes digitales, discursos y terminologías, relaciones interpersonales y TIC, compras y ventas a través de la web, TIC y tiempo, TIC y espacio y cuerpo y TIC.

\section{Nativos digitales-inmigrantes digitales}

Las TIC han cambiado universalmente la forma de interactuar en las relaciones humanas (Ortega, Rey y Sánchez, 2012; Wilson, Gosling y Graham, 2012), convirtiéndose en una herramienta y un medio para las redes sociales. Esto se evidencia con mayor frecuencia entre los jóvenes, la llamada generación de nativos digitales (Prensky, 2001; Bennett, Maton y Kervin, 2008), niños y jóvenes que desde el comienzo de sus vidas han sido socializados para utilizar las tecnologías digitales.

Actualmente los niños vienen con una capacidad tecnológica incluida desde que nacen; ellos comienzan con el proceso de repetir el aprendizaje. En cambio, las personas de cierta edad, a veces se restringen al aprendizaje de esas tecnologías, pensando que su momento de aprendizaje ya pasó y que no tienen esa capacidad. (Narrativa digital de HU1F)

Para mí, los jóvenes de todas las épocas han tenido la capacidad mental de asimilar todo el conocimiento que está a su alrededor. Estoy de acuerdo con lo de nativos e inmigrantes, ya que, por ejemplo, los jóvenes de los 80 por decirlo así, nacieron no con las tecnologías sino con los libros, ellos profundizaron más en lo que era la lectura física. Pero ahora, desde los 90 y el 2000 en adelante, ya nacieron con la tecnología al lado; por eso, desde pequeños son bastante curiosos o perspicaces con el manejo de estas herramientas. (Relato de HU1A en un grupo focal) 
Lo de nativos o inmigrantes digitales depende del contacto que tengas con las tecnologías; a veces los adultos no se desenvuelven tan bien porque no han tenido el suficiente contacto con ellas, que quizás tiene uno desde niño. (Relato de MU2A en un grupo focal)

En Colombia ha aumentado el uso de internet y de herramientas como smartphones; de acuerdo al Ministerio de Tecnologías de la Información y las Comunicaciones (Mintic), el número de equipos que tienen los colombianos para conectarse a internet de banda ancha aumentó 15 puntos entre 2015 y 2016: "mientras en 2015, por cada 100 colombianos había 54,5 terminales, en el 2016 la cifra subió a 69,55\%" (Mintic, 2016, p. 36). Estas cifras nos dan cuenta del impacto que están teniendo las TIC en las personas, donde cada vez es más necesario el uso de dispositivos y estar conectados.

Se podría decir que los estudiantes hoy parecen ser nativos del lenguaje digital desde la primera infancia y con cierto impacto en su pensamiento, y sus procesos de comunicación y socialización. Aunque las tecnologías digitales facilitan la creación de comunidades de interés y el uso de otras tecnologías, hay tendencias preocupantes como su uso adictivo, el procesamiento fraccionado de la información y las brechas digitales.

\section{Discursos, terminologías}

El uso diario de diferentes tipos de aplicaciones ha generado en los jóvenes universitarios de Montería, y en los jóvenes en general, nuevos discursos y nuevas terminologías; los más comunes son twittear, facebookear, whatsappear, trinar, tomarse selfies, etiquetar. Para Feixa (2006), esto es el resultado de las interacciones comunicativas de una subcultura juvenil, puesto que son nuevos términos que se han añadido al argot de los jóvenes y dependen de la aplicación que estén usando.

Otro término es "fara", es quien tenga una foto en Facebook, 500, 700, 1000 o más "likes" o "me gusta", entonces 
los grupos de jóvenes compiten por quienes sean más fara. Fara viene de farándula y se da sobre todo en los sectores más populares. (Relato de HU1LD en un grupo focal)

Es muy común que los jóvenes sigan a algunos youtubers, como se evidencia en los siguientes apartados:

Los youtuber influyen mucho, demasiado, mueven mucha, últimamente me sucedió que pensaba que solo era de cosas muy triviales, cosas que le sucedían, y hay muchos que te comentan por ejemplo sobre libros, hay de todo, también los hay para juego personas normales como nosotros, ellos tienen su cámara, su computador y su formato para editar y se paran ahí a grabar y ponen un tema y le sacan conclusiones y dan opiniones y a la vez le colocan música, efectos visuales. (Relato de MU2A en un grupo focal)

Los youtubers, por ejemplo, como Sebastián Villalobos, nacieron de ese auge que hay de las redes sociales. De Chile hay un chico muy famoso que muestra cosas que le suceden a los jóvenes y el canal en Youtuber es "Soy German". Martina la peligrosa, con unos videos sobre "Mamas de telenovela", "Diccionario cordobés", ella fue teniendo esa acogida con las personas. (Relato de HU1LD en un grupo focal)

Se denominan youtubers las personas que graban videos y los suben al portal web YouTube. Normalmente, suben videos enfocados en los intereses de jóvenes y adolescentes, que son los que más visitan este tipo de páginas. YouTube es considerado como un mediador de la cultura popular en el ciberespacio (Renó, 2007). A través de YouTube, el usuario, después de registrarse con algunos datos personales, puede publicar cualquier video, de su autoría o no, que tenga una duración máxima de 10 minutos y un tamaño de archivo de un máximo de $100 \mathrm{Mb}$. Para que un video de esta configuración pueda ser producido, es necesario solo una cámara 
fotográfica digital o un aparato celular con videocámara y un programa de edición. Estas facilidades permiten que cada día aumente la producción de contenidos digitales, como son los videos.

Con los faras y youtubers se reflejan nuevos grupos que se han formado con las redes sociales y otras herramientas, donde se muestran formas de ser y estar en el mundo actual; excelentes espacios para comprender nuevas formas de subjetivaciones que se están produciendo.

\section{Relaciones interpersonales y TIC}

La mundialización de la cultura ha puesto el énfasis en las TIC, reduciendo la comunicación a dichos artefactos que tienen la capacidad de ponernos en contacto a distancia (Mattelart, 2006). Son relevantes los modos de interacción que se promueven con los dispositivos, en particular el teléfono móvil y la forma como estos se introducen en la cotidianidad de los usuarios (Muñoz, 2007).

Se están perdiendo las conversaciones con diferentes personas en un mismo lugar, es muy incómodo el simple hecho de hablar con alguien y que no le presten atención por atender los dispositivos móviles. (Relato de la historia de vida de HU1A)

La socialización en línea por parte de los jóvenes ha sido uno de los campos más investigados en las últimas décadas (Shove y Pantzar, 2005; Erazo y Muñoz, 2007; Bennett, Maton y Kervin, 2008; Aguilar y Said, 2010; Ortega, Rey y Sánchez, 2012; Amador, 2013; Gardner y Davis, 2014; Sánchez, Muñoz y Ortega, 2015; Abdel-Aziz, Abdel-Salam y El-Sayad, 2016; Fuente, 2017; Suárez, Vélez y Londoño, 2018). Los estudios realizados han revelado que el uso de internet junto con las redes sociales y aplicaciones de mensajería instantánea están teniendo un impacto fundamental en las relaciones interpersonales de los adolescentes, en los que la búsqueda de la compañía de los iguales y la cercanía son de fácil acceso (Callén y Tirado, 2006; Collins, Welsh y Furman, 2009; 
Valkenburg, Sumter y Peter, 2011; Álvarez y Vélez, 2015; Przepiorka, Blachnio y Cudo, 2019).

Dentro de las relaciones interpersonales, las de pareja tienen un papel fundamental entre los jóvenes:

Había una chica que me gustaba mucho, y tan pronto hice una cuenta en Facebook, le solicité amistad y ella aceptó. Yo era muy tímido, pero al estar en Facebook, le dije a la chica que me gustaba, y ella me aceptó de novio. (Relato de la historia de vida de HU1JJ)

Las relaciones de pareja adolescentes satisfacen las necesidades de intimidad, afiliación, compañerismo y unión sexual, lo que contribuye al desarrollo de importantes competencias sociales y emocionales. A medida que los adolescentes y los jóvenes utilizan las nuevas tecnologías en su vida cotidiana, se podría esperar que estas dinámicas de relaciones adolescentes se expresen a través de dichas tecnologías (Collins, Welsh, y Furman, 2009; Furman y Buhrmester, 2009).

\section{Compras y ventas a través de la web}

Con el aumento de las tecnologías de telecomunicaciones, las actividades en el ámbito público comenzaron a disminuir, ya que la información se convirtió en fácilmente accesible desde el hogar, a través de internet (Yunis, Tarhini y Kassar, 2018). Esto también permitió el ascenso de las compras electrónicas, mientras que las conversaciones y debates parcialmente emigraron a foros web (Yang, Ren y Adomavicius, 2018). La internet se ha identificado como una nueva forma de espacio público, un ágora de la información (Abdel-Aziz, Abdel-Salam y El-Sayad, 2016).

Las compras y ventas a través de internet se están convirtiendo en actividades comunes, como pedir domicilios y comer en la universidad con aplicaciones desde su teléfono celular, vender a través de páginas de Facebook. A continuación algunos apartados al respecto: 
Entre las páginas que más visito está Facebook, en la cual uso las páginas de "Montería Vende", "Mercado negro Unicórdoba"; con la información de estas páginas ayudo a algunos compañeros cuando necesitan algo, como celulares, arreglar un computador, un perro, y verifico el contacto que ofrecen y los comentarios recibidos, para arreglar encuentros, observar el producto y proceder a hacer las compras. (Relato de la historia de vida de HU1A)

Compro libros en línea, en las librerías de la Nacional o la Lerner. Si el libro no lo venden en Colombia, algunas veces lo ha comprado por Amazon. (Relato de MU2MC en un grupo focal)

Acá vale la pena mencionar que el comportamiento de compra en línea depende de la edad, la orientación de los padres, las redes sociales y los compañeros (Gómez, 2016). El comercio electrónico en Colombia pasa por un momento de crecimiento muy importante; en el país las ventas online crecieron un $64 \%$ en el 2017, y se esperaba que, en América Latina, para el 2019 alcanzaran los \$ 85 000 millones de dólares. Las cuatro categorías de mayor demanda son: entretenimiento, moda, tecnología y mejora del hogar (Dinero, 2017).

Se afirma que internet es un agente importante en el proceso de socialización de los consumidores (Thaichon, 2017), por lo cual dentro de las ciencias sociales es necesario empezar a investigar a fondo qué cambios se están generando en las personas en general con las nuevas formas de comprar, en especial en línea.

\section{TIC y tiempo}

El uso de las TIC se caracteriza a menudo por que los usuarios se quedan hasta altas horas de la noche, lo que aparentemente se relaciona con niveles más bajos de rendimiento académico (Austin y Totaro, 2011). Al respecto: 
En un día normal de universidad, después de que me levanto, organizo el bolso dependiendo del horario y los cursos que voy a trabajar. Voy a las clases de la mañana, luego en la universidad me siento en el computador a investigar, o a hacer trabajos; por lo general mi hobby es programar; entonces me dedico a hacer programas o a investigar cómo hacerlo; descargo programas y juegos. Cuando cierro el computador, me voy para mi pensión y sigo estudiando, aplicando lo que investigué; cuando termino - ya que si no termino no me duermo-, ya descanso y duermo. (Narrativa digital de HUCA)

Las TIC son un recurso valioso, aunque usarlas en exceso puede generar dependencia por parte de los jóvenes. Se plantea la necesidad de fomentar medidas de protección que favorezcan el desarrollo de los individuos, que estén orientadas hacia estilos de vida saludables, así como a normalizar el uso de tales dispositivos, pues el tiempo que los jóvenes permanezcan con dichos dispositivos es un factor determinante en el riesgo de dependencia (Fuente, 2017).

\section{TIC y espacio}

Los continuos avances tecnológicos y la revolución en los teléfonos móviles, internet inalámbrico, Bluetooth, GPS y todas sus aplicaciones asociadas han influido en la forma en que las personas interactúan entre sí y con el espacio físico que los rodea (Pineda y Tamayo, 2016). Se plantean interrogantes sobre el impacto de estas tecnologías en el uso y el acto de comunicación en el espacio público y cómo pueden los planificadores urbanos crear un nuevo tipo de espacio público habitable, atractivo, seguro y sostenible con una infraestructura de alta tecnología de fluidos (Brandtzæg, Heim y Karahasanović, 2011).

Se han roto las barreras de tiempo, espacio y distancia con este tipo de herramientas y tecnologías; al tiempo que se implementan más tecnologías, las personas avanzan, en el sentido de que se tiene la facilidad de encontrar 
diferentes cosas, información y herramientas. (Relato de HU1S en un grupo focal)

No importa con quien esté, siempre estoy usando mi celular o tablet; solo lo dejo cuando mis padres me regañan en la mesa, o mi novia me dice que estoy hablando. Algunas veces me doy cuenta que es incómodo y que tal vez me esté perdiendo de algún momento. (Narrativa digital de HU1H)

Las TIC/TAC están permeando los estilos de vida y dan forma a la realización de actividades diarias. A través de los relatos de las historias de vida y de los grupos focales, al igual que en las narrativas digitales, se hace evidente que hay dispositivos tecnológicos y aplicaciones que se han convertido en fundamentales para el desarrollo de las actividades diarias, lo que ha facilitado las acciones en el tiempo-espacio.

Los jóvenes universitarios monterianos usan las TIC como una fuente de expresión; por ello, el uso de memes, redes sociales y blogs es común para dar a conocer lo que sienten sobre diferentes temas de interés, con lo cual se evidencia que en el establecimiento de nuevas redes sociales a través del entorno virtual se fortalecen las redes existentes o se reconstruyen las redes que se habían perdido por limitantes espaciales o geográficos.

La cercanía física ha cedido su lugar al uso de mediaciones tecnológicas que la suplen con creces, y la vehiculizan a través de herramientas que reconfiguran el escenario relacional. Los sujetos se relacionan a través de sus imágenes, de sus proyecciones consignadas en las herramientas digitales, abriendo de esta forma un escenario en el cual la psiquis individual y colectiva se transforma, dando paso al relacionamiento atravesado por la tecnología.

\section{Cuerpo y TIC}

Durante las interacciones diarias de cuerpo a cuerpo, las capacidades para codificar y decodificar las expresiones emocionales 
juegan un papel vital en la creación de vínculos sociales (Plested, Vallejo y Londoño, 2004), y al igual que la producción de los intercambios culturales influyen en las relaciones y la comunicación de significados (Bartenieff y Lewis, 2013). En cuanto a los cambios en el cuerpo

Desde que empecé a manejar un computador he aumentado de peso, el tiempo que le invierto a estar pegado en el computador, es quizás el que debería usar en hacer deporte, de jugar fútbol, de trotar, de salir a caminar, y ese ha sido uno de los cambios que he tenido a nivel físico. (Relato de historia de vida de HU1A)

A pesar de los enormes avances que se han hecho en el sector de las TIC, ha habido una escasez de investigación sobre los factores relacionados con el uso de las TIC por parte de los adolescentes para la interacción social. Un número significativo de jóvenes se sienten seguros de utilizar las TIC y creen que estas pueden ayudarles a aprender y lograr sus objetivos (Watty, McKay y Ngo, 2016). No obstante, investigaciones recientes también muestran que algunos jóvenes están reportando malestar físico por el uso de las TIC, como ojos doloridos y dolor en el cuello y los hombros. En ese sentido, las molestias se perciben como consecuencias negativas de las TIC (Scherer y Hatlevik, 2017).

El relacionamiento pasa a ser mental, deja de ser físico, el contacto físico desaparece, dando paso al intercambio emocional que lo suple parcialmente; el surgimiento de portales web para el relacionamiento afectivo, amistoso y amoroso es un signo de ello. En el caso de los jóvenes de Montería, parte de la cultura caribe, todo apunta a que prevalecerá el uso y la costumbre tendencial impuesta por estas herramientas digitales. El aislamiento en el mundo individual da lugar al nacimiento de nuevos escenarios, y eventualmente crea las condiciones para la ruptura de lazos colectivos que, como los deportes, la danza, los juegos de mesa, constituyen parte del patrimonio cultural local. 


\section{Conclusiones}

El avance de las tecnologías de la información y comunicación (TIC) con su ampliación al aprendizaje (TAC) ha permitido desarrollar espacios virtuales (redes sociales, blog, wikis, entre otros) que han ganado espacios relevantes en la interacción de los jóvenes $y$, por tanto, estos han vistos permeadas sus subjetividades y adquirido con ello nuevas expresiones. De allí la relevancia de investigar esta temática desde las ciencias sociales, en pro no solo de identificar cambios y posibles causas, sino de poder develar los tránsitos dados por estos jóvenes a través de las TIC y conocer algunos de sus efectos,

Por otro lado, hubo una apuesta metodológica cuyos resultados fueron positivos. Puesto que el trabajo a partir de la epistemología del sujeto conocido permitió darle relevancia a la opinión, las expectativas, las percepciones y los intereses de los jóvenes, lo que implica reconocer su capacidad para incidir en la transformación de sus propios contextos. Se reconoce a cada uno como un sujeto político, una subjetividad que construye poder y posibilita una mayor autonomía y capacidad de decisión sobre sus procesos educativos.

En cuanto a los hallazgos, es pertinente mencionar la emergencia del término faras, que aparece en el discurso de los estudiantes de ambas universidades, quienes lo mencionan en varias ocasiones y lo cargan connotativamente de una valoración positiva y, probablemente, deseable. Pues, al parecer, los jóvenes participantes consideran que necesitan mostrarse y tener la aprobación de la mayor cantidad de personas en las redes sociales. Una especie de jóvenes ciberhomogeneizados; en ellos, vestimenta, construcciones corpóreas y formas de relacionarse son similares. Lo anterior probablemente sea una de las causas del porqué los youtubers son tan relevantes para los jóvenes participantes dado que dichos youtubers han alcanzado un reconocimiento en los diferentes grupos etarios, agrupaciones $y$, en algunos casos, subculturas juveniles, proporcionando pautas sobre lo esperado, lo vivido y lo requerido 
en ese momento específico para ser reconocido, seguido y deseado.

En otras palabras, los faras y los youtubers, en los relatos de los jóvenes participantes, se manifiestan como réplicas agenciadas del mercado de las TIC, donde se evidencia el impacto de la globalización. Esta ha dado lugar a una homogeneización constante; entonces, los jóvenes ¿qué posibilidad tienen de subjetivarse?; posiblemente, esta sea limitada, si se entiende por subjetivizarse a quien reconoce que todo lo determina, pero trata de tomar distancia para criticar eso que lo determina.

Por otra parte, los sujetos de la educación contemporánea están permeados por las TIC en varios aspectos de su vida. Formas de ser, enseñar y aprender se confunden en los espacios de formación universitaria, con el resultado de que la mayoría de las veces los docentes quedan relegados por estas transformaciones, siendo superados por los estudiantes en apropiación de la información y el manejo de las mediaciones mencionadas.

Y esto se da gracias a que el joven que usa las TIC está introducido en este sistema informacional que le da oportunidades para desarrollar sus objetivos de vida. El riesgo de la disolución de los sujetos por la cultura de masas — que se ha expandido con las TIC/ TAC - no está solo en el uso del medio. El riesgo está en lo que se es ahora, porque ya se está supeditado a lo que se presenta en las pantallas, bien sea del televisor, del computador, de las tabletas, de los dispositivos móviles.

En esa línea de reflexión se puede pensar en el surgimiento de una forma cultural de subjetivación de los jóvenes que ven y usan las TIC como su forma de vida, de relacionarse y de ser, a tal punto que se asemeja a la cibercultura. Los ciudadanos del espacio aldeano coexisten con las formas ciberculturales de comunicación y relación.

Finalmente, la particularidad de un sujeto juvenil digital se puede desarrollar, promover, identificar y promocionar a través de 
contenidos digitales que muestren lo que son como personas -individuos no homogeneizados-, generando la visibilidad simbólica de una cultura local, teniendo en cuenta la hibridación de las culturas que los identifica. Por ello resalta la importancia de generar contenidos que visibilicen lo que representa ser de un contexto particular, ya que si no se afianza la visibilidad simbólica, lo que se dirá es que todos son homogéneos.

\section{Referencias bibliográficas}

Abdel-Aziz, A, Abdel-Salam, H. y El-Sayad, Z. (2016). The role of ICTs in creating the new social public place of the digital era. Alexandria Engineering Journal, 55(1), 487-493. DOI: http://doi.org/10.1016/j. aej.2015.12.019

Aguilar, D. y Said, E. (2010). Identidad y subjetividad en las redes sociales virtuales: caso de Facebook. Zona Próxima: Revista del Instituto de Estudios en Educación, 12. Recuperado de https://www.academia. edu/4102419/AGUILAR_and_SAID_2010._Identidad_y_subjetividad_ en_las_redes_sociales_virtuales_caso_de_Facebook

Álvarez, G. y Blanquicett, J. (2015). Percepciones de los docentes rurales sobre las TIC en sus prácticas pedagógicas. Ciencia, Docencia y Tecnología, 26(51), 371-394. Recuperado de http://www.redalyc.org/ articulo.oa?id=14542676016

Álvarez, G. y Vélez, C. (2014). ¿Qué se ha hecho sobre las TIC en educación superior y sobre la relación jóvenes y TIC? Revisión a investigaciones realizadas en América, Europa y Asia. REIRE: Revista d'Innovació i Recerca en Educación, 7(2), 28-52. Recuperado de http://revistes.ub.edu/index. $\mathrm{php/REIRE/issue/view/938}$

Álvarez, G. y Vélez, C. (2015). Configuración de subjetividades en los jóvenes universitarios sobre las tecnologías de la información, la comunicación y del aprendizaje (TIC/TAC). Itinerario Educativo, 
29(65), 223-236. Recuperado de http://revistas.usb.edu.co/index.php/ Itinerario/article/view/1710/1485

Amador, J. C. (2013). Aprendizaje transmedia en la era de la convergencia cultural interactiva. Educación y Ciudad, 25, 11-24. Recuperado de https://dialnet.unirioja.es/servlet/articulo?codigo $=5705093$

Arias, Ó., Londoño, D. L., Betancourt, R. A. y Restrepo, J. H. (2017). Theoretical foundations on relationship between $\mathrm{KM}$ and territorial planning. En Proceedings of the 18th European Conference on Knowledge Management (pp. 1277-1281). Reading: Academic Conferences and Publishing International. Recuperado de https://search.proquest. com/openview/bc426276888c3a1c86cc2feb280083f1/1 ?pqorigsite $=$ gscholar $\&$ cbl $=1796412$

Austin, W. y Totaro, M. (2011). Gender differences in the effects of internet usage on high school absenteeism. The Journal of SocioEconomics, 40, pp. 192-198. Recuperado de https://ideas.repec.org/a/ eee/soceco/v40y2011i2p192-198.html

Baron, N. (2008). Always on: Language in an online and mobile world. Oxford: Oxford University Press.

Bartenieff, I., y Lewis, D. (2013). Body movement: Coping with the environment. Nueva York: Routledge.

Baudrillard, J. (1989). El otro por sí mismo. Signo y Pensamiento, 8(14), 71-77. Recuperado de https://revistas.javeriana.edu.co/index.php/ signoypensamiento/article/view/5728

Bauman, Z. (2017). Vida líquida. Bogotá: Planeta.

Bennett, S., Maton, K. y Kervin, L. (2008). The 'digital natives' debate: A critical review of the evidence. British Journal of Educational Technology, 39, 775-786. DOI: http://dx.doi.org/10.1111/j.1467-8535.2007.00793.x

Boisvert, R. F. e Irwin, M. J. (2006). Plagiarism on the rise. Communications of the ACM, 49(6), 23-24. DOI: http://doi.acm. 
$\operatorname{org} / 10.1145 / 1132469.1132487$

Brandtzæg, P., Heim, J. y Karahasanović, A. (2011). Understanding the new digital divide - a typology of internet users in Europe. International Journal of Human-Computer Studies, 69(3), 123-138. DOI: http://dx.doi. org/10.1016/j.ijhcs.2010.11.004

Callén, B., y Tirado, F. (2006). Biodatas y dividuos: la transformación de la biopolítica en la era de la información. En F. Tirado y $\mathrm{M}$. Domènech (eds.), Lo social y lo virtual: nuevas formas de control y transformación social (pp. 28-45). Barcelona: UOC.

Campos, M. y Mújica, A. (2008). El análisis de contenido: una forma de abordaje metodológico. Laurus, 14, 129-144. Recuperado de http:// www.redalyc.org/articulo.oa?id=76111892008

Cardona, Á. M., Valencia, E., Duque, J. H. y Londoño, D. A. (2015). Construcción de los planes de vida de los jóvenes: una experiencia de investigación en la vereda La Doctora, Sabaneta (Antioquia). Aletheia: Revista de Desarrollo Humano, Educativo y Social Contemporáneo, 7(2), 90-113. Recuperado de http://www.scielo.org.co/scielo.php?pid=S214503662015000200005\&script=sci_abstract\&tlng=en

Castells, M. et al. (1986). El desafío tecnológico: España y las nuevas tecnologías. Buenos Aires: Alianza.

Collins, W., Welsh, P. y Furman, W. (2009). Adolescent romantic relationships. Annual Review of Psychology, 60, 631-652. Recuperado de DOI: http://dx.doi.org/10.1146/annurev.psych.60.110707.163459

Curran, J., Fenton, N. y Freedman, D. (2016). Misunderstanding the internet. Nueva York: Routledge.

DeLanda, M. (2006). A new philosophy of society: Assemblage theory and social complexity. Londres: A\&C Black.

Dede, C. (2000). Aprendiendo con tecnología. Buenos Aires: Paidós. 
Deleuze, G. (2002). Empirismo y subjetividad. Barcelona: Gedisa.

Dinero. (2017). Así crecen las ventas "online" en América Latina. Recuperado de http://www.dinero.com/edicion-impresa/lagrafica/articulo/crecimiento-del-comercio-electronico-en-americalatina/247678

Duggan, M., Ellison, N., Lampe, C. Lenhart, A. y Madden, M. (2015). Social media update 2014. Pew Research Center. Recuperado de http:// www.pewinternet.org/2015/01/09/social-media-update-2014/

Duriau, F., Reger, G. y Pfarrer, J. (2007). A content analysis of the content analysis literature in organization studies: Research themes. Organizational Research Methods, 10(1), 5-34. Recuperado de https:// journals.sagepub.com/doi/abs/10.1177/1094428106289252

Emerson, L. (2014). Reading writing interfaces: From the digital to the bookbound. Mineápolis: University of Minnesota Press.

Erazo, E. y Muñoz, G. (2007). Las mediaciones tecnológicas en los procesos de subjetivación juvenil: interacciones en Pereira y Dosquebradas, Colombia. Revista Latinoamericana de Ciencias Sociales, Niñez y Juventud, 2(5), 723-754. Recuperado de http://biblioteca.clacso.edu.ar/gsdl/ collect/co/co-001/index/assoc/D8587.dir/ArtEdgarDiegoErazoCaicedo. pdf

Ertmer, P. (2005). Teacher pedagogical beliefs: The final frontier in our quest for technology integration? Educational Technology Research and Development, 53(4), 25-39. Recuperado de https://eric. ed.gov/?id=EJ732691

Feixa, C. (2006). Generación XX: teorías sobre la juventud en la era contemporánea. Revista Latinoamericana de Ciencias Sociales, Niñez y Juventud, 4(2), 21-45. Recuperado de http://www.scielo.org.co/scielo. php?pid=S1692-715X2006000200002\&script=sci_arttext\&tlng=pt

Foucault, M. (1988). El sujeto y el poder. Revista Mexicana de Sociología, 50(3), 3-20. Recuperado de https://www.jstor.org/stable/3540551 
Fuente, R. de la (2017). ICTs and teenage students: Problematic usage or dependence. Procedia - Social and Behavioral Sciences, 237, 230-236. DOI: https://doi.org/10.1016/j.sbspro.2017.02.068

Furman, W. y Buhrmester, D. (2009). Methods and measures: The network of relationships inventory: Behavioral systems version. International Journal of Behavioral Development, 33(5), 470-478. Recuperado de DOI: http://dx.doi.org/10.1177/0165025409342634

García Canclini, N. (1997). Culturas híbridasyestrategias comunicacionales. Estudios sobre las Culturas Contemporáneas, 3(5), 109-128. Recuperado de https://www.redalyc.org/articulo.oa?id=31600507

Gardner, H. y Davis, K. (2014). La generación App: cómo los jóvenes gestionan su identidad, su privacidad y su imaginación en el mundo digital. Barcelona: Paidós.

Genlott, A. y Grönlund, A. (2016). Closing the gaps - Improving literacy and mathematics by ict-enhanced collaboration. Computers \& Education. 99, 68-80. DOI: https://doi.org/doi:10.1016/j.compedu.2016.04.004

Giraldo, F. L., Zúñiga, S. E., Londoño, D. A. y Sánchez, L. M. (2018). La lectura en la apropiación de la ciencia y la tecnológica. Revista Latinoamericana de Estudios Educativos (Colombia), 14(2), 158178. Recuperado de https://www.redalyc.org/pdf/1341/Resumenes/ Resumen_134157078008_1.pdf

Gisbert, M. (1992). Technology based training: formador de formadores en la dimensión ocupacional [documento policopiado]. Universidad Rovira i Virgili, Tarragona, España.

Goktas, Y. y Demirel, T. (2012). Blog-enhanced ICT courses: Examining their effects on prospective teachers' ICT competencies and perceptions. Computers \& Education, 58(3), 908-917. Recuperado de http://www. sciencedirect.com/science/article/pii/S0360131511002740

Gómez, J. A. (2016). Colombian consumer's decision making online process: An ex-post-fact study. Avances en Psicología Latinoamericana, 
34(2), 5. Recuperado de https://dialnet.unirioja.es/servlet/ articulo?codigo $=5456389$

González, A. (2000). Medición, experimentación y descubrimiento en las ciencias sociales. Caracas: Universidad Central de Venezuela.

Gutiérrez, G. y Orozco, J. C. (2007). Políticas tecnológicas en un escenario de gestión de conocimiento en educación. Revista Iberoamericana de Educación, 45. Recuperado de https://rieoei.org/historico/documentos/

Hartley, J. (2008). Repurposing literacy: The uses of Richard Hoggart for creative education. En S. Owen (ed.), Richard Hoggart and Cultural Studies (pp. 137-157). Nueva York: Palgrave Macmillan.

Hattangdi, A. y Ghosh, A. (2008). Enhancing the quality and accessibility of higher education through the use of information and communication technology. Recuperado de https://www.semanticscholar.org/paper/ Enhancing-the-quality-and-accessibility-of-higher-Hattangdi-Ghosh/02c 7b0093f49c9284ee23878d276aa2b85f4800a

Jaramillo, P. y Ruiz, M. (2009). Un caso de integración de TIC que no agrega valor al aprendizaje. Revista Latinoamericana de Ciencias Sociales, Niñez y Juventud, 7(1), 267-287. Recuperado de http://www.scielo.org. co/scielo.php?script=sci_arttext\&pid=S1692-715X2009000100011

Jorgensen, D. L. (2015). Participant observation. En R. Scott, S. Kosslyn y M. Buchmann (eds.), Emerging Trends in the Social and Behavioral Sciences: An Interdisciplinary, Searchable, and Linkable Resource, 1-15. Nueva York: John Wiley \& Sons. Recuperado de https://onlinelibrary. wiley.com/doi/abs/10.1002/9781118900772.etrds0247

Latour, B. (2007). Nunca fuimos modernos: ensayo de antropología simétrica. Buenos Aires: Siglo XXI.

Lee, C. S. y Ma, L. (2012). News sharing in social media: The effect of gratifications and prior experience. Computers in Human Behavior, 28(2), 331-339. Recuperado de https://www.sciencedirect.com/science/ article/pii/S074756321100210X 
Londoño, D., Olave, J., Jaime, J. y Losada, N. (2018). Lógicas, enfoques y epistemologías sobre educación y pedagogía. En Centro de Estudios Avanzados en Niñez y Juventud, Universidad de Manizales (Cinde), Educación y pedagogía: trayectos recorridos (96-155). Manizales: Universidad de Manizales.

Lozano, R. (2011). Las 'TIC/TAC': de las tecnologías de la información y comunicación a las tecnologías del aprendizaje y del conocimiento. Anuario ThinkEPI, 5, 45-47. Recuperado de https://recyt.fecyt.es/index. php/ThinkEPI/article/view/30465

Lupton, D. A. (2014). 'Feeling better connected': Academics' use of social media. Canberra: News \& Media Research Centre.

Mattelart, A. (2006). Diversidad cultural y mundialización. Barcelona: Paidós.

Mandavilli, A. (2011). Trial by Twitter. Nature, 469, 286-287. Recuperado de http://www.axeleratio.com/news/trial_by_twitter_nature2011.pdf

Martín-Barbero, J. (2011). Prólogo. En M. Díaz et al., Tribu capital (pp. 9-13). Bogotá: Fundación Fahrenheit 451.

Massaro, D. W. (1993). Information processing models: Microscopes of the mind. Annual Review of Psychology, 44(1), 383-425. DOI: http:// dx.doi.org/10.1146/annurev.ps.44.020193.002123

Mintic (Ministerio de Tecnologías de la Información y las Comunicaciones). (2016). Diseño y medición indicador Terminales por cada 100 habitantes: en el marco del Plan Nacional de Desarrollo 2014-2018. Recuperado de http://www.mintic.gov.co/portal/604/articles-51641_recurso_1.pdf

Muñoz, G. (2007). La comunicación en los mundos de vida juveniles. Revista Latinoamericana de Ciencias Sociales. Niñez y Juventud, 1(5), 283-308. Recuperado de http://www.scielo.org.co/pdf/rlcs/v5n1/ v5n1a09.pdf

Ortega, R., Rey, R. del y Sánchez, V. (2012). Nuevas dimensiones de 
la convivencia escolar y juvenil. Ciberconducta y relaciones en la red: ciberconvivencia. Madrid: Ministerio de Educación, Cultura y Deporte.

Papastergiou, M., Gerodimos, V. y Antoniou, P. (2011). Multimedia blogging in physical education: Effects on student knowledge and ICT self-efficacy. Computers \& Education, 57, 1998-2010. DOI: https:/doi. org/10.1016/j.compedu.2011.05.006

Pié Balaguer, A. (2014). Por una corporeidad postmoderna: nuevos tránsitos sociales y educativos para la interdependencia. Barcelona: UOC.

Pineda, J. E. y Tamayo, L. H. (2016). E-moderating and e-tivities: The implementation of a workshop to develop online teaching skills in inservice teachers. Profile Issues in Teachers' Professional Development, 18(1), 97-114. Recuperado de http://www.scielo.org.co/scielo. php?script=sci_arttext $\&$ pid $=$ S1657-07902016000100006

Plested, M. C., Vallejo, G. y Londoño, D. (2004). Harmonization of non verbal communication in diving meets. 9th Annual Congress European College of Sport Science. Clermont Ferrand, 95.

Prat, Q. y Camerino, O. (2012). Las tecnologías del aprendizaje y el conocimiento (TAC) en la educación física, la WebQuest como recurso didáctico. Apunts: Educación Física y Deportes, 109, 44-53. Recuperado de https://www.researchgate.net/publication/236843381_ Las_tecnologias_del_aprendizaje_y_el_conocimiento_TAC_en_la_ educacion_fisica_la_WebQuest_como_recurso_didactico

Prensky, M. (2001). Digital natives, digital immigrants: part 1. On the Horizon, 9(5), 1-6. Recuperado de https://www.emeraldinsight.com/ doi/abs/10.1108/10748120110424816

Przepiorka, A., Blachnio, A. y Cudo, A. (2019). The role of depression, personality, and future time perspective in internet addiction in adolescents and emerging adults. Psychiatry Research, 272, 340-348. Recuperado de https://www.sciencedirect.com/science/article/pii/ S0165178118311247 
Reig, D. (2012). Conociendo la creatividad para potenciarla. Recuperado de http://www.dreig.eu/caparazon/2012/04/23/creatividad/

Renó, D. (2007). YouTube, el mediador de la cultura popular en el ciberespacio. Revista Latina de Comunicación Social, 10(62), 1-5. Recuperado de http://4www.redalyc.org/articulo.oa?id=81906217

Roy, A. (2012). Subjects of risk: Technologies of gender in the making of millennial modernity. Public Culture, 24(1), 131-155. Recuperado de https://read.dukeupress.edu/public-culture/article-abstract/24/1\%20 (66)/131/31022

Sánchez, V., Muñoz, N. y Ortega, R. (2015). "Cyberdating Q_A": An instrument to assess the quality of adolescent dating relationships in social networks. Computers in Human Behavior, 48, 78-86. Recuperado de https://www.researchgate.net/publication/272073825_"Cyberdatin g_Q_A"_An_instrument_to_assess_the_quality_of_adolescent_dating relationships_in_social_networks

Sandholtz, J. y Reilly, B. (2004). Teachers, not technicians: Rethinking technical expectations for teachers. The Teachers College Record, 106(3), 487-512. Recuperado de http://citeseerx.ist.psu.edu/viewdoc/downloa d?doi=10.1.1.550.833\&rep=rep1\&type $=p d f$

Scanlon, P. M. (2003). Student online plagiarism: How do we respond? College Teaching, 51(4), 161-166. DOI: https://doi. org/10.1080/87567550309596432

Scherer, R. y Hatlevik, O. (2017). "Sore eyes and distracted" or "excited and confident"? - The role of perceived negative consequences of using ICT for perceived usefulness and self-efficacy. Computers \& Education, 115, 188-200. DOI: https://doi.org/10.1016/j.compedu.2017.08.003

Shove, E. y Pantzar, M. (2005). Consumers, producers and practices: Understanding the invention and reinvention of Nordic walking. Journal of Consumer Culture, 5, 43-64. DOI: https://doi. org/10.1177/1469540505049846 
Stewart, D. W. y Shamdasani, P. N. (2014). Focus groups: Theory and practice (Vol. 20). Londres: Sage.

Suárez, P. A., Vélez, M., y Londoño, D. A. (2018). Las herramientas y recursos digitales para mejorar los niveles de literacidad y el rendimiento académico de los estudiantes de primaria. Revista Virtual Universidad Católica del Norte, 54, 184-198. Recuperado de http://revistavirtual. ucn.edu.co/index.php/RevistaUCN/article/view/990

Taipale, S. (2015). Bodily dimensions of reading and writing practices on paper and digitally. Telematics and Informatics, 32(4), 766-775. Recuperado de http://www.sciencedirect.com/science/article/pii/ S0736585315000325

Thaichon, P. (2017). Consumer socialization process: The role of age in children's online shopping behavior. Journal of Retailing and Consumer Services, 34, 38-47. Recuperado de https://ideas.repec.org/a/eee/ joreco/v34y2017icp38-47.html

Valkenburg, P., Sumter, S. y Peter, J. (2011). Gender differences in online and offline self-disclosure in pre-adolescence and adolescence. British Journal of Developmental Psychology, 29(2), 253-269. DOI: http://dx.doi. org/10.1348/2044-835x.002001

Vasilachis, I. (2006). Estrategias de Investigación cualitativa. Barcelona: Gedisa.

Villalobos, J. L., Flórez, G. A. y Londoño, D. A. (2017). Relación maestro - alcance del logro: una mirada a los procesos de enseñanza en la escuela. Entramado, 13(1), 186-196. Recuperado de http://www. scielo.org.co/scielo.php?pid=S1900-38032017000100186\&script=sci_ abstract\&tlng=en

Vivancos, J. (Febrero de 2009). Ensenyament de les Ciències amb suport TAC. Recuperado de http://ticotac.blogspot.com.co/2011/02/ ensenyament-de-les-ciencies-amb-suport.html

Watty, K., McKay, J. y Ngo, L. (2016). Innovators or inhibitors? Accounting 
faculty resistance to new educational technologies in higher education. Journal of Accounting Education, 36. DOI: https://doi.org/10.1016/j. jaccedu.2016.03.003

Whelan, E., Teigland, R., Vaast, E. y Butler, B. (2016). Expanding the horizons of digital social networks: Mixing big trace datasets with qualitative approaches. Information and Organization, 26(1), 1-12. Recuperado de https://aran.library. nuigalway.ie/handle/10379/7208

Wilson, R., Gosling, S. y Graham, L. (2012). A review of Facebook research in the social sciences. Perspectives on Psychological Science, 7(3), 203220. Recuperado de http://dx.doi.org/10.1177/1745691612442904

Yang, M., Ren, Y. C. y Adomavicius, G. (2018). Understanding usergenerated content and customer engagement on Facebook business pages. Information Systems Research, Forthcoming, 18-82. Recuperado de https://papers.ssrn.com/sol3/papers.cfm?abstract_id=3260294

Yin, R. (1984). Estudio de caso: diseño y métodos. Newbury Park: Sage.

Yunis, M., Tarhini, A. y Kassar, A. (2018). The role of ICT and innovation in enhancing organizational performance: The catalysing effect of corporate entrepreneurship. Journal of Business Research, 88, 344-356. Recuperado de https://www.sciencedirect.com/science/article/abs/pii/ S0148296317305246 